\title{
Phase I study of ipatasertib as a single agent and in combination with abiraterone plus prednisolone in Japanese patients with advanced solid tumors
}

\author{
Toshihiko Doi ${ }^{1}(1) \cdot$ Yutaka Fujiwara $^{2} \cdot$ Nobuaki Matsubara $^{3} \cdot$ Junichi Tomomatsu $^{4}(1) \cdot$ Satoru Iwasa $^{2} \cdot$ Akari Tanaka $^{5} \oplus$. \\ Chihiro Endo-Tsukude ${ }^{6}\left(\mathbb{D} \cdot\right.$ Shintaro Nakagawa $^{7} \cdot$ Shunji Takahashi $^{4}(\mathbb{0}$
}

Received: 14 January 2019 / Accepted: 31 May 2019 / Published online: 21 June 2019

(c) The Author(s) 2019

\begin{abstract}
Purpose Ipatasertib is a selective inhibitor of Akt, a frequently activated protein kinase in human cancers. The current study assessed the safety, tolerability, and pharmacokinetics of ipatasertib in Japanese patients with solid tumors.

Methods This was a phase I, open-label, $3+3$ dose-escalation study conducted in two stages. In stage I, Japanese patients with solid tumors were administered ipatasertib 200, 400, or $600 \mathrm{mg}$ /day for 21 days of a 28 -day cycle. In stage II, Japanese patients with castration-resistant prostate cancer were administered ipatasertib 200 or $400 \mathrm{mg} / \mathrm{day}$ in combination with abiraterone and prednisolone in 28-day cycles. Dose-limiting toxicity (DLT) was assessed at each dose before enrolling patients at a higher dose; DLT was used to determine the maximum tolerated dose (MTD) and maximum administered dose (MAD). Pharmacokinetic parameters were assessed after a single dose and at steady state.

Results Fifteen patients were enrolled in Stage I and six in Stage II. The ipatasertib MTD was $600 \mathrm{mg}$ as monotherapy and MAD was $400 \mathrm{mg}$ in combination with abiraterone and prednisolone. Ipatasertib plasma exposure was dose proportional across the dose range, and was not markedly affected by concurrent administration of abiraterone plus prednisolone. Stable disease (SD) was observed in eight patients treated with ipatasertib monotherapy (53.3\%); four patients had SD and one had complete response with ipatasertib plus abiraterone and prednisolone.

Conclusions Ipatasertib, at the monotherapy MTD of $600 \mathrm{mg} / \mathrm{day}$ and MAD of $400 \mathrm{mg} / \mathrm{day}$ in combination with abiraterone and prednisolone, was safe and tolerable in Japanese patients with solid tumors.
\end{abstract}

Keywords Dose-limiting toxicity $\cdot$ Ipatasertib $\cdot$ Pharmacokinetics $\cdot$ Prostate cancer $\cdot$ Akt inhibitor $\cdot$ PTEN

Electronic supplementary material The online version of this article (https://doi.org/10.1007/s00280-019-03882-7) contains supplementary material, which is available to authorized users.

Toshihiko Doi

tdoi@east.ncc.go.jp

1 Department of Experimental Therapeutics, National Cancer Center Hospital East, 6-5-1 Kashiwanoha, Kashiwa-shi, Chiba-ken 277-8577, Japan

2 Department of Experimental Therapeutics, National Cancer Center Hospital, Tokyo, Japan

3 Department of Breast and Medical Oncology, National Cancer Center Hospital East, Kashiwa, Japan

\section{Introduction}

The phosphatidylinositol 3-kinase (PI3K)/Akt/mammalian target of rapamycin (mTOR) signaling pathway is a key regulator of cellular responses to stress [1]. The tumor microenvironment is inherently stressful, with

4 Department of Medical Oncology, The Cancer Institute Hospital of Japanese Foundation for Cancer Research, Tokyo, Japan

5 Clinical Science and Strategy Department, Chugai Pharmaceutical Co., Ltd, Tokyo, Japan

6 Clinical Pharmacology Department, Chugai Pharmaceutical Co., Ltd, Tokyo, Japan

7 Clinical Information and Intelligence Department, Chugai Pharmaceutical Co., Ltd., Tokyo, Japan 
poor oxygenation, low $\mathrm{pH}$, and limited nutrient supply [1]. It is, therefore, unsurprising that this pathway plays a central role in the development and potentiation of cancer $[1,2]$. Activation of this pathway by mutations of the PIK3CA gene or loss of tumor suppressor phosphatase and tensin homolog (PTEN) protein expression promotes tumor growth and proliferation $[3,4]$. Serine/threonine kinase Akt (protein kinase B) plays an important role in the PI3K/Akt/mTOR pathway, and abnormally activated Akt is commonly seen in cancer [2,5], including metastatic castration-resistant prostate cancer (mCRPC) [6, 7]. Furthermore, non-clinical data suggest that reciprocal crosstalk between the androgen receptor and PI3K/Akt/ mTOR pathways is present in PTEN-loss mCRPC. Specifically, activation of the PI3K/Akt/mTOR pathway is associated with repressed androgen signaling, and inhibition of the PI3K/Akt/mTOR pathway restores androgen receptor signaling in PTEN-deficient prostate cells [8]. This suggests that combined inhibition of the androgen receptor and PI3K/Akt/mTOR pathways may result in measurable decline of tumor cell viability and more durable clinical benefit.

The central role of the PI3K/Akt/mTOR pathway in the oncogenic process has led to the development of cancer treatments targeting this pathway. For example, drugs that target the PI3K/Akt/mTOR pathway have shown activity in a range of cancers, including renal cell carcinoma [9] and triple-negative breast cancer (TNBC) [10], where conventional anti-cancer therapies have failed. However, most of the drugs that target PI3K/Akt/mTOR have shown limited activity as monotherapy, and there is greater potential for these drugs when administered in combination therapy [6, $11,12]$

Ipatasertib is a highly selective small-molecule inhibitor of Akt (Akt1, Akt2, and Akt3) [13-15], and is in development as a single agent and in combination with other therapies for the treatment of cancers in which activation of the $\mathrm{PI} 3 \mathrm{~K} / \mathrm{Akt} / \mathrm{mTOR}$ pathway is involved in tumor growth or therapeutic resistance $[16,17]$. Results of a randomized, double-blind phase II study of ipatasertib in combination with abiraterone and prednisone/prednisolone showed trends towards improved radiographic progression-free survival (PFS) and overall survival (OS) compared with placebo in patients with mCRPC who had a PTEN loss [11]. The treatment was well tolerated [11]. Similarly, in patients with TNBC, the randomized, double-blind phase II study (LOTUS) reported longer PFS with the combination of ipatasertib plus paclitaxel than with placebo plus paclitaxel, indicating the benefits of ipatasertib in this patient population [18].

The current phase I dose-escalation study was undertaken to investigate the safety, tolerability, and pharmacokinetics of ipatasertib alone and in combination with abiraterone + prednisolone for Japanese patients with advanced or recurrent/refractory solid tumors.

\section{Materials and methods}

\section{Study design}

This was a phase I, open-label, multicenter, $3+3$ dose-escalation study (JapicCTI-152,910) conducted at three centers in Japan. The study consisted of two stages. Stage I was designed to determine the maximum tolerated dose (MTD) and maximum administered dose (MAD) of ipatasertib monotherapy in Japanese patients with advanced or recurrent solid tumors, by investigating the safety, tolerability, and pharmacokinetics of ipatasertib in this population. Stage II determined the safety, tolerability, pharmacokinetics, and MTD/MAD of ipatasertib in combination with abiraterone and prednisolone in Japanese patients with CRPC.

The study protocol was approved by the institutional review boards of all participating centers and the study was conducted in accordance with the Declaration of Helsinki, Good Clinical Practice, and the Law for Ensuring the Quality, Efficacy, and Safety of Drugs and Medical Devices (paragraph 3 of article 14 and article 80-2).

All study participants provided written informed consent before entering the study.

\section{Patients}

Patients were included in the study if they were aged $\geq 20$ years with a histologically or cytologically confirmed, advanced or recurrent/refractory solid tumor (Stage I), or CRPC refractory to $\geq 1$ type of hormone therapy with serum testosterone levels of $<50 \mathrm{ng} / \mathrm{dL}$ and who were not candidates for docetaxel or in whom docetaxel was ineffective (Stage II). In addition, patients were required to have an Eastern Cooperative Oncology Group performance status (ECOG PS) of 0 or 1; a life expectancy of $\geq 12$ weeks after enrollment; lesion(s) that could be assessed by diagnostic imaging; major organ functioning within the required limits and sufficient cardiac function; a history of completing surgery, radiotherapy, chemotherapy, immunosuppressive therapy or treatment with other investigational drugs $\geq 4$ weeks before the study; or blood transfusion/hematopoietic factor products, endocrine therapy or immunotherapy $\geq 2$ weeks before the study.

Major exclusion criteria were hypersensitivity to hydroxypropyl methylcellulose (an excipient of ipatasertib); inability to take oral drugs or the presence of gastrointestinal issues that may interfere with drug absorption; meningeal or central nervous system (CNS) metastasis requiring treatment; previous adverse event 
(AE; grade $\geq 3$ ) with an investigational product targeting Akt; diabetes mellitus requiring insulin; or an autoimmune disease or hypercalcemia requiring treatment. Additional exclusion criteria in Stage II were hypersensitivity to abiraterone or prednisolone, and a history of adrenal insufficiency or hyperaldosteronism. A complete list of inclusion and exclusion criteria is shown in Online Resource 1.

\section{Treatments}

The study design and ipatasertib administration protocols are summarized in Fig. 1 and Online Resource 2, respectively.

In Stage I, patients received ipatasertib orally at escalating doses (200 mg, $400 \mathrm{mg}$, and $600 \mathrm{mg}$ ). First, they received a single dose on Day 1 followed by an off-treatment period. Subsequently, they received that dose of ipatasertib once daily for 21 days, followed by 7 days off, in 28-day cycles. The doses used were the same as the doses in the previous phase I study of ipatasertib [17].

In Stage II, patients received ipatasertib orally $(200 \mathrm{mg}$ and $400 \mathrm{mg}$ once daily for 28 days), followed by abiraterone (1000 mg once daily) and prednisolone (5 mg twice daily). This was the same dose as used in the previous phase II study of ipatasertib [11]. The dose escalation strategy used for the two treatment stages is shown in Online Resource 3.

The treatments were continued until progressive disease (PD), dose-limiting toxicity (DLT), or withdrawal of informed consent.

Concomitant administration of the following agents was prohibited during the study: anti-tumor drugs, prophylactic treatments to prevent AEs including granulocyte colonystimulating factors, St. John's wort, grapefruit, long-term systemic corticosteroids (except prednisolone administered in Stage II), other investigational or unapproved drugs, and drugs that prolong QT interval (Stage I).

\section{Study outcomes}

The primary objectives of this study were to determine the safety, tolerability, and pharmacokinetics of ipatasertib alone and in combination with abiraterone and prednisolone. Safety and tolerability were assessed by the occurrence of AEs and DLTs, and DLT was used to determine MTD and MAD. The severity of AEs was graded according to National Cancer Institute Common Terminology Criteria for Adverse Events (NCI CTCAE), version 4.03 [19].

DLTs were defined as the occurrence of AEs during the evaluation period for which a causal relationship with ipatasertib could not be ruled out, and which met the treatment discontinuation criteria or required drug suspension during

Fig. 1 Study design

Stage I

(advanced or recurrent solid tumors)
Stage II

(castration-resistant prostate cancer)

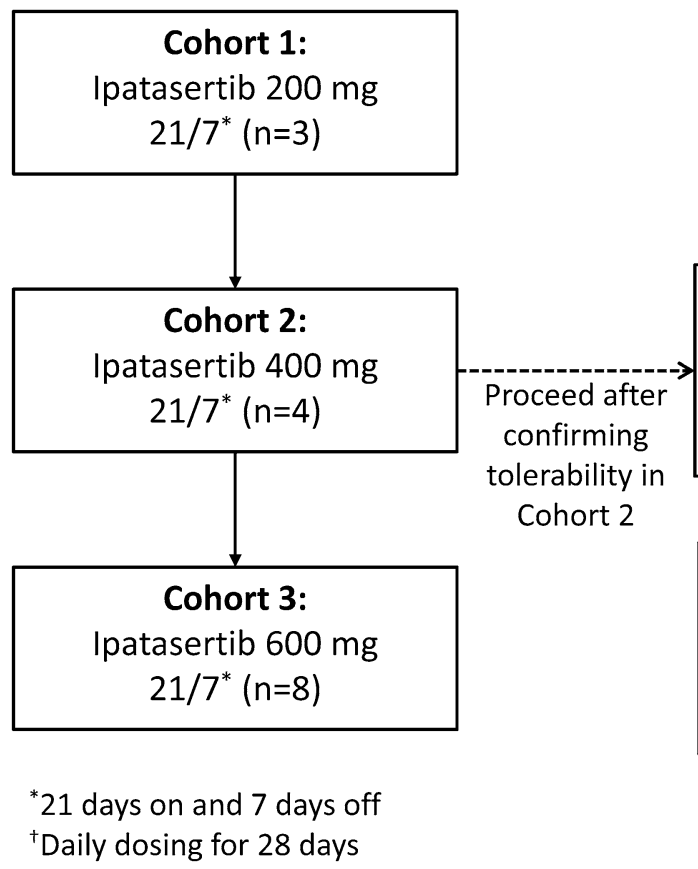

Cohort A:

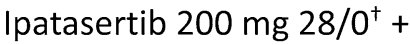
abiraterone + prednisolone $(n=3)$

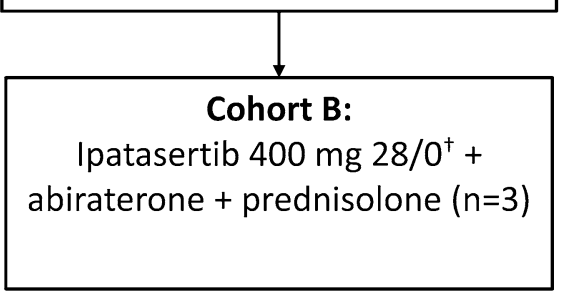

${ }^{\dagger}$ Daily dosing for 28 days 
the evaluation period. The DLT observation period for Stage I was from Day 1 of Cycle 0 to before administration on Day 1 of Cycle 2, and for Stage II, it was from Day 1 of Cycle 1 to before administration on Day 1 of Cycle 2. A complete list of potential DLTs considered in the study is included in Online Resource 4 and contains grade 4 neutropenia for $\geq 5$ days; febrile neutropenia; grade 3 thrombocytopenia requiring platelet transfusions or grade 4 thrombocytopenia; grade $\geq 4$ anemia; grade $\geq 3$ non-hematologic toxicity (excluding transient electrolyte abnormalities). The occurrence of a DLT at any given dose determined whether or not investigators would proceed to the next dose cohort. DLTs were used to determine the MTD of ipatasertib alone and in combination with abiraterone and prednisolone, defined as the highest dose at which $<33 \%$ of patients experienced a DLT. The MAD of ipatasertib alone was determined to be $600 \mathrm{mg}$ if no patients experienced a DLT in Cohort 3 , and that of ipatasertib in combination with abiraterone and prednisolone was determined to be $400 \mathrm{mg}$ if no patients experienced a DLT in Cohort B.

Pharmacokinetic parameters $\left(t_{\max }, C_{\max }, \mathrm{AUC}_{0-24}, t_{1 / 2}\right)$ for ipatasertib and its metabolite (G-037720) following a single dose (Stage I: Cycle 0, Day 1; Stage II: Cycle 1, Day 1) and repeated doses (Stage I: Cycle 1, Day 8; Stage II: Cycle 1, Day 15) were calculated using plasma drug concentration-time data. Serial blood samples were taken for $72 \mathrm{~h}$ after a single dose in Cycle 0 and on Day 8 in Cycle 1 during Stage I, and on Day 1 and Day 15 of Cycle 1 in Stage II (Online Resource 5).

Concentrations of ipatasertib and G-037720 were determined using a validated liquid chromatography-tandem mass spectrometry analytical procedure, with a lower limit of quantification of $0.500 \mathrm{ng} / \mathrm{mL}$ for both ipatasertib and G-037720. The accumulation ratio was calculated using the formula: [ $\mathrm{AUC}_{0-24}$ at steady state]/[ $\mathrm{AUC}_{0-24}$ following single dose].

The secondary objective of the study was to determine the preliminary efficacy of ipatasertib in both stages of the study. All tumor lesions were assessed using the Response Evaluation Criteria in Solid Tumors (RECIST) version 1.1 [20].

An exploratory objective of this study was to determine the relationship between tumor response and PTEN expression, and PI3K pathway gene mutation and amplification. $P I K 3 C A$ and $A k t 1$ mutation/amplification were detected in tumor tissue samples collected prior to study entry (archival samples) using a Semiconductor DNA sequencer and Ion AmpliSeq ${ }^{\mathrm{TM}}$ Cancer Hotspot Panel, version 2 (Thermo Fisher Scientific; Waltham, MA, USA). Copy number variations (CNVs) were detected in the Akt1 and PIK3CA genes, and were reported if CNV confidence value was $\geq 20$. Single nucleotide polymorphisms (SNPs) were reported if they had a frequency of $\geq 1$, coverage of $\geq 500$, and were located in a known hotspot (allele source). PTEN expression was analyzed in formalin-fixed, paraffin-embedded tissue samples by immunohistochemistry (IHC) using the VENTANA OptiView DAB IHC Detection Kit on the automated BenchMark ULTRA platform (Ventana Medical Systems; Tucson, AZ, USA) with the PTEN (SP218) rabbit monoclonal antibody assay (Spring Biosciences; Pleasanton, CA, USA) [21]. Once acceptable internal controls had been met, PTEN was considered to be intact if the specimen contained $>50 \%$ of viable malignant cells with any specific cytoplasmic stain intensity, and was considered to be lost if $\geq 50 \%$ of viable malignant cells had no specific cytoplasmic staining [21]. Nuclear staining of viable malignant cells was disregarded.

\section{Statistical analysis}

The planned sample size for the study was 15-30 patients in total, 9-18 patients in Stage I (3-6 per cohort) and an additional 6-12 patients in Stage II (3-6 per cohort). The safety analysis set included all patients who received $\geq 1$ dose of the study drug, and the DLT population included all patients from the safety analysis set who were evaluable for DLTs. The full analysis set included all patients who received $\geq 1$ dose of study drug and who subsequently underwent $\geq 1$ efficacy assessment.

The calculation of pharmacokinetic parameters was performed using WinNonlin Ver 6.4 (Pharsight Corporation, NC, USA), and data aggregation was performed using SAS, version 9 (SAS Institute Inc., NC, USA).

\section{Results}

\section{Patients}

The study was conducted at three centers between 29 May 2015 and 24 August 2017. Overall, 21 patients were enrolled, 15 in Stage I and 6 in Stage II (Table 1). Patients enrolled in Stage I had a median age of 58.0 years (range 35-76) and were mostly male (53.3\%); the majority of patients (80\%) had an ECOG PS of 0 (Table 1). In Stage II, patients had a median age of 70.5 years (range 45-77); all patients were male (100\%) and most (83.3\%) had an ECOG PS of 0 (Table 1). All patients in Stage II had received prior systemic therapies, including chemotherapy in four patients (66.7\%) and abiraterone or enzalutamide in five (83.3\%).

\section{Safety}

Ipatasertib was well tolerated at doses up to $600 \mathrm{mg}$ as monotherapy in Stage I and up to $400 \mathrm{mg}$ as combination therapy in Stage II. At least one AE was experienced by all patients, most commonly diarrhea and nausea (Table 2). 
Table 1 Baseline characteristics of patients included in the study $(N=21)$

\begin{tabular}{|c|c|c|c|c|c|c|c|}
\hline & \multicolumn{4}{|l|}{ Stage I } & \multicolumn{3}{|l|}{ Stage II } \\
\hline & $\begin{array}{l}\text { Ipatasertib } \\
200 \mathrm{mg} \\
(n=3)\end{array}$ & $\begin{array}{l}\text { Ipatasertib } \\
400 \mathrm{mg} \\
(n=4)\end{array}$ & $\begin{array}{l}\text { Ipatasertib } \\
600 \mathrm{mg} \\
(n=8)\end{array}$ & $\begin{array}{l}\text { Total } \\
(n=15)\end{array}$ & $\begin{array}{l}\text { Ipatasertib } \\
200 \mathrm{mg}+\mathrm{ABI}+\mathrm{PRE} \\
(n=3)\end{array}$ & $\begin{array}{l}\text { Ipatasertib } \\
400 \mathrm{mg}+\mathrm{ABI}+\mathrm{PRE} \\
(n=3)\end{array}$ & $\begin{array}{l}\text { Total } \\
(n=6)\end{array}$ \\
\hline \multicolumn{8}{|l|}{ Sex, $n(\%)$} \\
\hline Male & $1(33.3)$ & $1(25.0)$ & $6(75.0)$ & $8(53.3)$ & $3(100.0)$ & $3(100.0)$ & $6(100.0)$ \\
\hline Age $^{\mathrm{a}}$, years & $37.0(35-58)$ & $59.5(54-68)$ & $66.0(49-76)$ & $58.0(35-76)$ & $71.0(62-74)$ & $70.0(45-77)$ & $70.5(45-77)$ \\
\hline Weight $^{\mathrm{a}}, \mathrm{kg}$ & $\begin{array}{l}49.00 \\
\quad(47.2-57.1)\end{array}$ & $\begin{array}{l}58.85 \\
\quad(52.3-68.9)\end{array}$ & $\begin{array}{l}59.10 \\
\quad(47.3-73.9)\end{array}$ & $\begin{array}{l}58.05 \\
\quad(47.2-73.9)\end{array}$ & $\begin{array}{l}62.90 \\
\quad(59.3-83.8)\end{array}$ & $\begin{array}{l}68.80 \\
\quad(68.8-84.2)\end{array}$ & $\begin{array}{l}68.80 \\
\quad(59.3-84.2)\end{array}$ \\
\hline \multicolumn{8}{|c|}{ ECOG PS, $n(\%)$} \\
\hline 0 & $2(66.7)$ & $3(75.0)$ & $7(87.5)$ & $12(80.0)$ & $3(100.0)$ & $2(66.7)$ & $5(83.3)$ \\
\hline 1 & $1(33.3)$ & $1(25.0)$ & $1(12.5)$ & $3(20.0)$ & $0(0.0)$ & $1(33.3)$ & $1(16.7)$ \\
\hline \multicolumn{8}{|c|}{ Number of prior systemic therapies, $n(\%)$} \\
\hline 2 & 0 & $1(25.0)$ & $1(12.5)$ & $2(13.3)$ & 0 & 0 & 0 \\
\hline$\geq 3$ & $3(100.0)$ & $3(75.0)$ & $7(87.5)$ & $13(86.7)$ & $3(100.0)$ & $3(100.0)$ & $6(100.0)$ \\
\hline $\mathrm{PSA}^{\mathrm{a}}, \mu \mathrm{g} / \mathrm{L}$ & - & - & - & - & $\begin{array}{l}5.3 \\
\quad(4.5-202.2)\end{array}$ & $\begin{array}{l}96.5 \\
\quad(43.6-236.5)\end{array}$ & $\begin{array}{l}70.1 \\
\quad(4.5-236.5)\end{array}$ \\
\hline \multicolumn{8}{|c|}{ Type of cancer, $n(\%)$} \\
\hline Bladder & 0 & 0 & $3(37.5)^{*}$ & $3(20.0)$ & 0 & 0 & 0 \\
\hline Cervical & 0 & 0 & $2(25.0)$ & $2(13.3)$ & 0 & 0 & 0 \\
\hline Colorectal & 0 & 0 & $1(12.5)$ & $1(6.7)$ & 0 & 0 & 0 \\
\hline CRPC & 0 & 0 & 0 & 0 & $3(100.0)$ & $3(100.0)$ & $6(100.0)$ \\
\hline $\begin{array}{l}\text { Duodenum } \\
\text { papilla }\end{array}$ & 0 & 0 & $1(12.5)$ & $1(6.7)$ & 0 & 0 & 0 \\
\hline Gastric & $1(33.3)$ & 0 & 0 & $1(6.7)$ & 0 & 0 & 0 \\
\hline GIST & 0 & 0 & $1(12.5)$ & $1(6.7)$ & 0 & 0 & 0 \\
\hline Liver & $1(33.3)$ & 0 & $1(12.5)^{*}$ & $2(13.3)$ & 0 & 0 & 0 \\
\hline Ovarian & $1(33.3)$ & $1(25.0)$ & 0 & $2(13.3)$ & 0 & 0 & 0 \\
\hline Peritoneal & 0 & $1(25.0)$ & 0 & $1(6.7)$ & 0 & 0 & 0 \\
\hline Ureteral & 0 & $1(25.0)$ & 0 & $1(6.7)$ & 0 & 0 & 0 \\
\hline Unknown & 0 & $1(25.0)$ & 0 & $1(6.7)$ & 0 & 0 & 0 \\
\hline \multicolumn{8}{|c|}{ Cancer histology, n (\%) } \\
\hline $\begin{array}{l}\text { Adenocarci- } \\
\text { noma }\end{array}$ & $2(66.7)$ & $3(75.0)$ & $2(25.0)$ & $7(46.7)$ & $3(100.0)$ & $3(100.0)$ & $6(100.0)$ \\
\hline GIST & 0 & 0 & $1(12.5)$ & $1(6.7)$ & 0 & 0 & 0 \\
\hline $\mathrm{HCC}$ & $1(33.3)$ & 0 & $1(12.5)^{*}$ & $2(13.3)$ & 0 & 0 & 0 \\
\hline $\mathrm{SCC}$ & 0 & 0 & $2(25.0)$ & $2(13.3)$ & 0 & 0 & 0 \\
\hline UC & 0 & $1(25.0)$ & $3(37.5)^{*}$ & $4(26.7)$ & 0 & 0 & 0 \\
\hline
\end{tabular}

$A B I$ abiraterone, $C R P C$ castration-resistant prostate cancer, ECOG PS Eastern Cooperative Oncology Group performance status, GIST gastrointestinal stromal tumor, $H C C$ hepatocellular carcinoma, PRE prednisolone, PSA prostate-specific antigen, SCC squamous cell carcinoma, UC urothelial carcinoma

*One patient had bladder and liver cancer (histologist UC and HCC, respectively)

${ }^{a}$ Median (range)

Grade 3 AEs developed in four patients treated with ipatasertib $600 \mathrm{mg}$ during Stage I. These events were nausea $(n=2)$, hyperglycemia $(n=2)$, diarrhea $(n=1)$, and colitis/ dehydration $(n=1)$. During Stage I, serious AEs (SAEs) were reported in one patient who developed grade 3 colitis that was considered related to study drug, accompanied by grade 3 dehydration that was considered unrelated to study drug; no SAEs occurred in Stage II of the study. The patient made a complete recovery after treatment discontinuation. Two patients developed grade 3 AEs during Stage II while receiving ipatasertib $400 \mathrm{mg}$ in combination therapy; these events were urticaria $(n=1)$ and anemia $(n=1)$. No grade 4 AEs or deaths occurred during either of the two stages. 
Table 2 Adverse events in Stage I and Stage II of the study $(N=21)$

\begin{tabular}{|c|c|c|c|c|c|c|c|}
\hline \multirow[t]{2}{*}{ AEs, $n(\%)$} & \multicolumn{4}{|l|}{ Stage I } & \multicolumn{3}{|l|}{ Stage II } \\
\hline & $\begin{array}{l}\text { Ipatasertib } \\
200 \mathrm{mg} \\
(n=3)\end{array}$ & $\begin{array}{l}\text { Ipatasertib } \\
400 \mathrm{mg} \\
(n=4)\end{array}$ & $\begin{array}{l}\text { Ipatasertib } \\
600 \mathrm{mg} \\
(n=8)\end{array}$ & $\begin{array}{l}\text { Total } \\
(n=15)\end{array}$ & $\begin{array}{l}\text { Ipatasertib } 200 \\
\mathrm{mg}+\mathrm{ABI}+\mathrm{PRE} \\
(n=3)\end{array}$ & $\begin{array}{l}\text { Ipatasertib } 400 \\
\mathrm{mg}+\mathrm{ABI}+\mathrm{PRE} \\
(n=3)\end{array}$ & $\begin{array}{l}\text { Total } \\
(n=6)\end{array}$ \\
\hline Any & 3 & 4 & 8 & $15(100)$ & 3 & 3 & $6(100)$ \\
\hline \multicolumn{8}{|l|}{ AEs reported in $\geq 2$ patients } \\
\hline Diarrhea & - & 3 & 7 & $10(66.7)$ & 2 & 3 & $5(83.3)$ \\
\hline Nausea & 1 & 2 & 7 & $10(66.7)$ & 3 & 3 & $6(100)$ \\
\hline Decreased appetite & - & 2 & 5 & $7(46.7)$ & - & 1 & $1(16.7)$ \\
\hline Vomiting & - & 1 & 4 & $5(33.3)$ & 1 & 1 & $2(33.3)$ \\
\hline Fatigue & 1 & 2 & 2 & $5(33.3)$ & - & 1 & $1(16.7)$ \\
\hline Hyperglycemia & - & - & 2 & $2(13.3)$ & - & - & - \\
\hline AST increased & - & - & 2 & $2(13.3)$ & - & - & - \\
\hline ALT increased & - & - & 2 & $2(13.3)$ & - & - & - \\
\hline Blood insulin increased & - & - & 2 & $2(13.3)$ & - & - & - \\
\hline $\begin{array}{l}\text { Blood creatinine } \\
\text { increased }\end{array}$ & - & 1 & 1 & $2(13.3)$ & - & - & - \\
\hline Glucose urine present & - & - & 2 & $2(13.3)$ & - & - & - \\
\hline Rash & 1 & - & 1 & $2(13.3)$ & - & 1 & $1(16.7)$ \\
\hline Back pain & 1 & 1 & - & $2(13.3)$ & - & - & - \\
\hline Diabetes mellitus & - & - & - & - & 2 & - & $2(33.3)$ \\
\hline Dysgeusia & - & - & 1 & $1(6.7)$ & - & 2 & $2(33.3)$ \\
\hline Dizziness & - & - & - & - & - & 2 & $2(33.3)$ \\
\hline
\end{tabular}

$A B I$ abiraterone, $A L T$ alanine aminotransferase, $A S T$ aspartate aminotransferase, $P R E$ prednisolone

In Stage I, 12 of 15 patients were evaluated for DLT; 3 patients (400 mg, $n=1 ; 600 \mathrm{mg}, n=2$ ) were not evaluable because they discontinued the study before the end of the evaluation period for reasons other than AEs (patient decision). No DLTs were reported with ipatasertib $200 \mathrm{mg}$ or $400 \mathrm{mg}$, and one patient on ipatasertib $600 \mathrm{mg}$ experienced grade 3 nausea, which required drug withdrawal for more than 6 days, during the DLT observation period. The MTD for ipatasertib was $600 \mathrm{mg} /$ day for 21 days of a 28-day cycle.

No DLTs developed during Stage II of the study. The MAD for ipatasertib was $400 \mathrm{mg} /$ day when used in combination with abiraterone and prednisolone in the 28-day cycle schedule.

\section{Pharmacokinetics}

The pharmacokinetic study population in Stage I consisted of 14 patients. Data from one patient in Stage I $(200 \mathrm{mg})$ were excluded from the pharmacokinetic analysis because this patient had a history of total surgical gastrectomy and lower esophagectomy, which could affect drug absorption.

Ipatasertib as a single agent was rapidly absorbed after oral administration. The $t_{\max }$ was reached at a median of 2.53-3.03 h after the first administration of ipatasertib at a dose of $200-600 \mathrm{mg}$. The geometric mean $t_{1 / 2}$ was between
18.8 and $24.3 \mathrm{~h}$ at these doses (Table 3). The plasma ipatasertib concentration reached steady state within 7 days after daily administration, with an accumulation ratio between 1.38 and 1.82 (Table 3). The plasma concentrations of ipatasertib increased proportionally with dose escalation in the dose range of 200-600 mg (Fig. 2a, b).

G-037720 was detected in plasma soon after the administration of a single dose of ipatasertib. Its median $t_{\max }$ was $3.00-3.05 \mathrm{~h}$, and geometric mean $t_{1 / 2}$ was $21.3-29.7 \mathrm{~h}$ after administration of ipatasertib 200-600 mg (Stage I, Cycle 0, Day 1). G-037720 was considered to be the main metabolite of ipatasertib, since the geometric mean of metabolite/parent $(\mathrm{M} / \mathrm{P})$ ratio of $\mathrm{AUC}_{0-\mathrm{inf}}$ after single administration of 200-600 mg ipatasertib was 0.426-0.884.

Abiraterone and prednisolone did not markedly affect the plasma concentration profile of ipatasertib. The plasma concentrations of ipatasertib increased with dose escalation (single and repeated doses; Fig. 2c, d). The geometric mean $\mathrm{AUC}_{0-24}$ following repeated doses of ipatasertib $400 \mathrm{mg}$ plus abiraterone and prednisolone (4970 h ng/mL, GCV 17.8\%; Stage II, Cycle 1, Day 15) was comparable to that observed following repeated doses of ipatasertib $400 \mathrm{mg}$ as a single agent (4870 h ng/mL, GCV 43.1\%; Stage I, Cycle 1, Day 8). However, the $\mathrm{AUC}_{0-24}$ for G-037720 was approximately twofold higher in patients receiving multiple doses of 
Table 3 Single dose and steady-state pharmacokinetic parameters of ipatasertib during the study

\begin{tabular}{|c|c|c|c|c|c|}
\hline \multirow{4}{*}{$\begin{array}{l}\text { Single dose pharma- } \\
\text { cokinetics }\end{array}$} & \multicolumn{3}{|l|}{ Stage I } & \multicolumn{2}{|l|}{ Stage II } \\
\hline & Ipatasertib $200 \mathrm{mg}$ & Ipatasertib $400 \mathrm{mg}$ & Ipatasertib $600 \mathrm{mg}$ & Ipatasertib $200 \mathrm{mg}$ & Ipatasertib $400 \mathrm{mg}$ \\
\hline & \multicolumn{3}{|l|}{ Cycle 0, Day 1} & \multicolumn{2}{|l|}{ Cycle 1, Day 1} \\
\hline & $(n=2)$ & $(n=4)$ & $(n=8)$ & $(n=3)$ & $(n=3)$ \\
\hline$C_{\max }{ }^{\mathrm{a}}, \mathrm{ng} / \mathrm{mL}$ & $151(3.75)$ & $456(36.6)$ & $953(36.0)$ & 214 (49.9) & $328(46.0)$ \\
\hline$T_{\max }^{\mathrm{b}}, \mathrm{h}$ & $2.53(1.98-3.08)$ & $3.03(1.02-4.07)$ & $2.57(0.52-4.00)$ & $0.97(0.95-3.98)$ & $3.97(3.90-4.02)$ \\
\hline$t_{1 / 2}^{\mathrm{c}}, \mathrm{h}$ & $24.3(23.7-24.8)$ & $18.8(17.0-21.1)$ & $21.5(16.2-33.9)$ & $7.34(7.23-7.45)$ & $\mathrm{NC}$ \\
\hline $\mathrm{AUC}_{0-24}{ }^{\mathrm{a}}, \mathrm{h} \mathrm{ng} / \mathrm{mL}$ & $805(30.7)$ & $4010(38.6)$ & $5930(33.1)$ & $1250(36.4)$ & $2940(29.6)$ \\
\hline \multirow{4}{*}{$\begin{array}{l}\text { Steady-state pharma- } \\
\text { cokinetics }\end{array}$} & \multicolumn{3}{|l|}{ Stage I } & \multicolumn{2}{|l|}{ Stage II } \\
\hline & Ipatasertib $200 \mathrm{mg}$ & Ipatasertib $400 \mathrm{mg}$ & Ipatasertib $600 \mathrm{mg}$ & Ipatasertib $200 \mathrm{mg}$ & Ipatasertib $400 \mathrm{mg}$ \\
\hline & \multicolumn{3}{|l|}{ Cycle 1, Day 8} & \multicolumn{2}{|l|}{ Cycle 1, Day 15} \\
\hline & $(n=2)$ & $(n=4)$ & $(n=7)$ & $(n=3)$ & $(n=3)$ \\
\hline$C_{\max }{ }^{\mathrm{a}}, \mathrm{ng} / \mathrm{mL}$ & $186(4.17)$ & $579(43.1)$ & $973(57.4)$ & $334(31.3)$ & $452(35.0)$ \\
\hline$T_{\max }^{\mathrm{b}}, \mathrm{h}$ & $1.46(0.97-1.95)$ & $1.48(0.93-4.00)$ & $1.97(0.47-3.03)$ & $1.98(1.95-2.02)$ & $3.97(3.87-4.03)$ \\
\hline$t_{1 / 2}^{\mathrm{c}}, \mathrm{h}$ & 7.69 (7.49-7.90) & $7.20(7.03-7.32)$ & $8.06(6.50-10.30)$ & $8.29(7.62-8.84)$ & $\mathrm{NC}$ \\
\hline $\mathrm{AUC}_{0-24}{ }^{\mathrm{a}}, \mathrm{h} \mathrm{ng} / \mathrm{mL}$ & $1210(23.5)$ & $4870(43.1)$ & $6510(57.6)$ & $2710(28.7)$ & $4970(17.8)$ \\
\hline Accumulation ratio & $1.82(3.58)$ & $1.43(9.41)$ & $1.38(51.7)$ & $2.16(10.0)$ & $1.69(11.8)$ \\
\hline
\end{tabular}

$A U C_{0-24}$ Area under concentration-time curve from 0 to $24 \mathrm{~h}, N C$ not calculated

${ }^{\mathrm{a}} \mathrm{Geometric}$ mean $(\% \mathrm{CV})$

${ }^{\mathrm{b}}$ Median (range)

${ }^{\mathrm{c}}$ Geometric mean (range)

ipatasertib $400 \mathrm{mg}$ plus abiraterone and prednisolone (Stage II, Cycle 1, Day 15) compared with patients receiving ipatasertib $400 \mathrm{mg}$ as a single agent [Stage I, Cycle 1, Day 8; 4540 (33.9) vs. 2230 (38.0) h ng/mL (GCV\%)].

\section{Efficacy}

During Stage I, efficacy was evaluated in 14 of 15 patients treated with ipatasertib. One patient discontinued the treatment before the post-treatment tumor assessment and was excluded from the efficacy evaluation. The best overall response was stable disease (SD) in eight patients and PD in six patients. The percentage change from baseline in target lesions is shown in Fig. 3a.

All six patients treated with ipatasertib during Stage II who were evaluable had a treatment history of more than four regimens for CRPC. One of these patients had a CR, four patients had SD, and one had PD. The percentage change from baseline in target lesions of three patients who had measurable lesions at screening is shown in Fig. 3b. Three patients were able to continue treatment for six cycles or more, despite the fact that two of them had a history of abiraterone and enzalutamide treatment.

\section{Gene alteration status}

A total of 15 tumor samples were evaluated for PTEN, $P I K 3 C A$, and Akt1. PTEN status was evaluable in seven patients, one of whom had PTEN loss with SD. PIK3CA mutations were detected in eight patients, five of whom had SD and one of whom was not evaluable. Tumor shrinkage $(-11.9 \%$, $-6.1 \%$ ) was observed in two patients (one with cervical cancer; one with peritoneal cancer) who had PIK3CA mutation in the helical domain (E542K or E545K) and/or amplification (Fig. 3c). PIK3CA amplification was detected in three patients, while Aktl mutation and amplification were not detected.

\section{Discussion}

This $3+3$ dose-escalation phase I study showed that ipatasertib was well tolerated, with a favorable safety profile when administered either alone (MTD, $600 \mathrm{mg} /$ day) or in combination with abiraterone and prednisolone (MAD, $400 \mathrm{mg} /$ day). The data also show that ipatasertib was rapidly absorbed after oral administration, and its plasma concentration profile is unaffected by concomitant administration of abiraterone and prednisolone. When used as monotherapy in patients with solid tumors, the best overall response with 

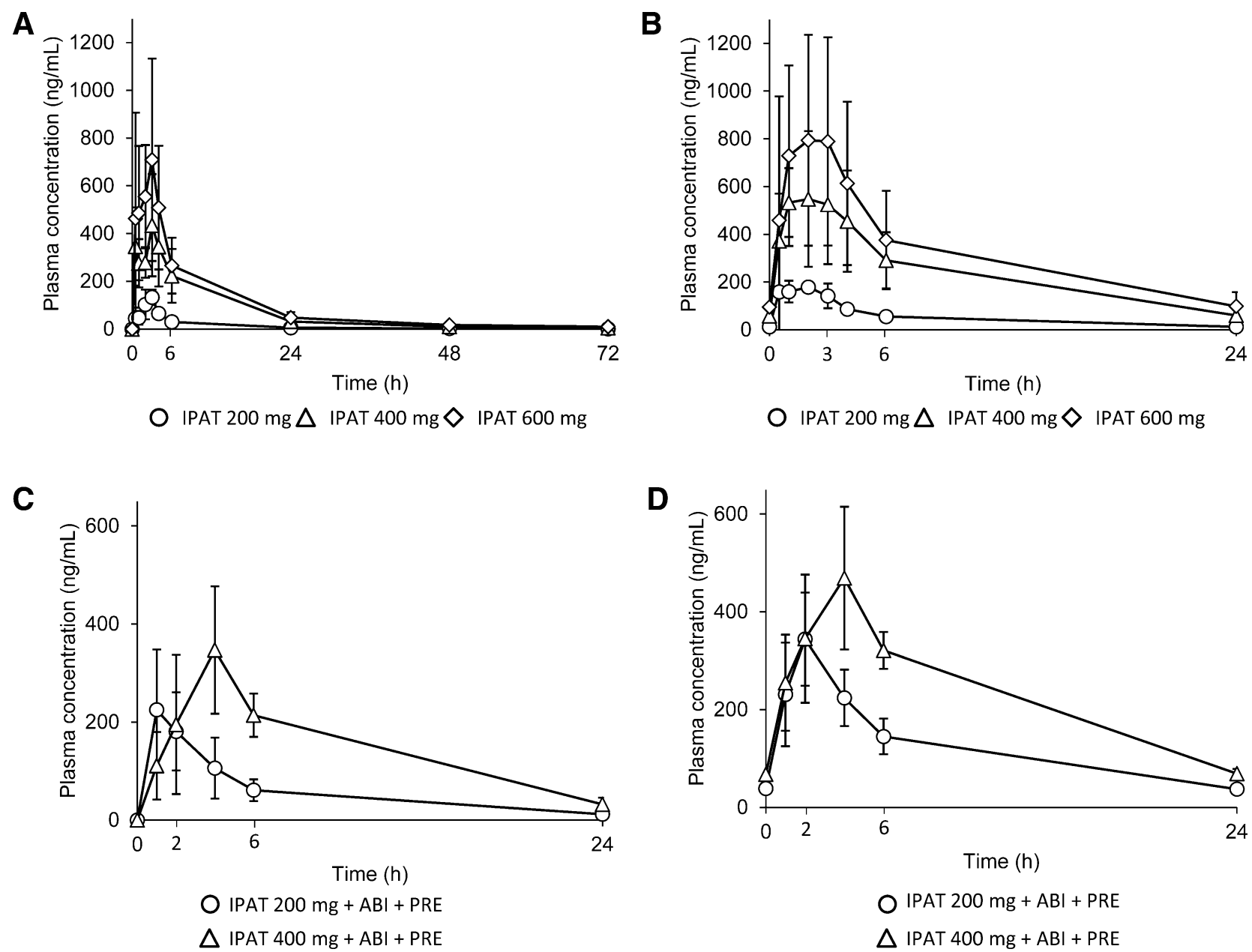

Fig. 2 Mean (standard deviation) plasma concentration of ipatasertib at steady state after single and repeated doses. a Stage I, single dose (Cycle 0, Day 1); b Stage I, repeated doses (Cycle 1, Day 8); c Stage

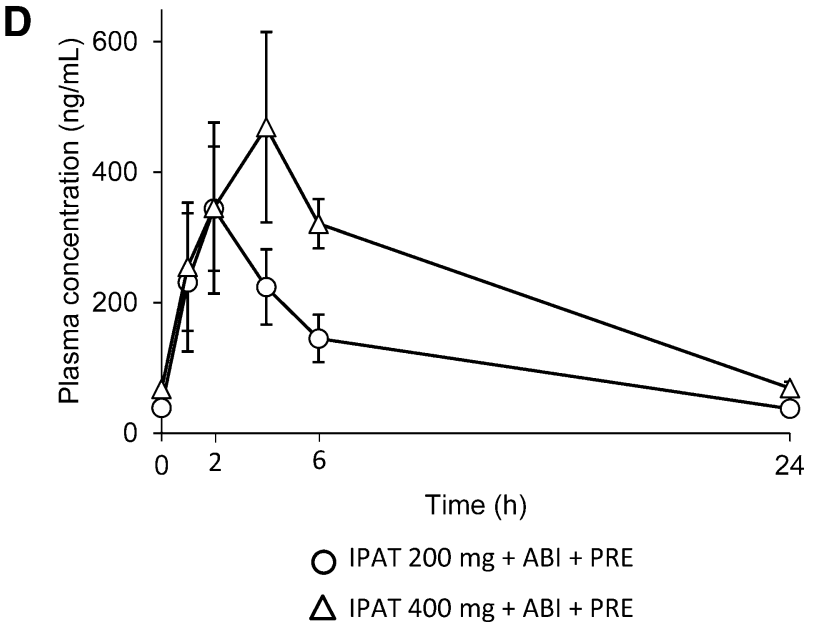

II, single dose (Cycle 1, Day 1); and d Stage II repeated doses (Cycle 1, Day 15). ABI, abiraterone; IPAT, ipatasertib; PRE, prednisolone

ipatasertib was SD in eight patients, while the best response with ipatasertib plus abiraterone and prednisolone was CR in one patient and SD in four patients.

During the present study, the most common AEs observed with ipatasertib monotherapy were diarrhea, nausea, decreased appetite, fatigue, and vomiting, and the most common AEs with combination therapy were nausea and diarrhea. These events were mostly grade 1 or 2 in severity. Grade 3 events developed in four patients with ipatasertib monotherapy (nausea, hyperglycemia, diarrhea, and colitis/ dehydration) and in two patients during combination therapy (anemia and urticaria). No patients developed grade 4 or 5 AEs during ipatasertib treatment as either monotherapy or in combination. The safety profile of ipatasertib as monotherapy or combination therapy in this study was consistent with what is expected of agents targeting the PI3K/Akt/ mTOR pathway and with the safety profile of ipatasertib observed in non-Japanese patients. The phase I and II studies

with ipatasertib in non-Japanese patients also reported diarrhea, nausea, and hyperglycemia as common AEs [11, 17].

The $\mathrm{AUC}_{0-24}$ and $C_{\max }$ for ipatasertib monotherapy were found to be dose dependent in the present study. The mean $\mathrm{AUC}_{0-24}$ of ipatasertib at steady state was approximately 0.7- to 1.5-fold of that reported in previous phase I study of ipatasertib [17]. Although the mean plasma exposures were higher, the plasma exposures in individual patients showed significant overlap, and the data may be confounded by the small number of patients evaluated for this comparison.

Combining ipatasertib with abiraterone and prednisolone in Stage II of the present study did not majorly affect the plasma concentration profile of ipatasertib. Although a twofold increase in $\mathrm{AUC}_{0-24}$ of the main metabolite of ipatasertib (G-037,720) was observed after repeated administration of the combination compared with ipatasertib monotherapy, G-037,720 is less active compared with ipatasertib and is expected to have limited anticancer activity. The exact 


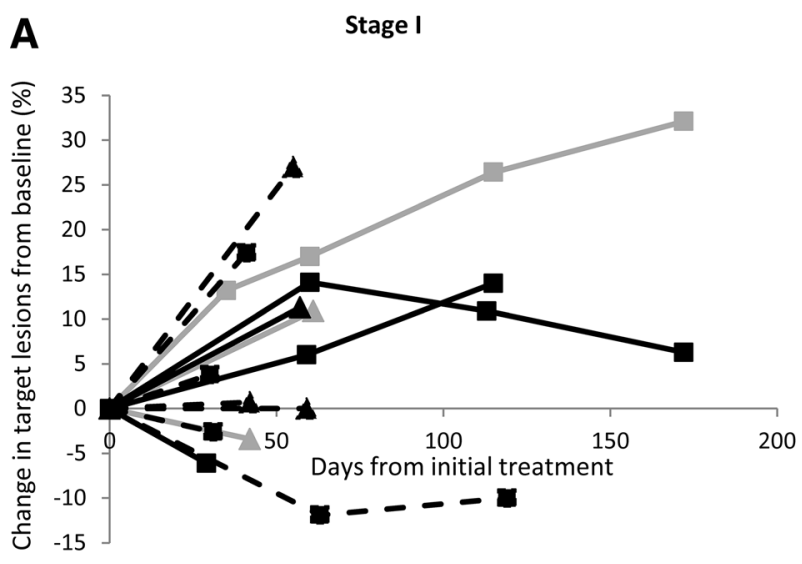

IPAT $200 \mathrm{mg}-$ IPAT $400 \mathrm{mg}--$ IPA $600 \mathrm{mg}$
Best overall response: $\triangle$ PD $\square$ SD

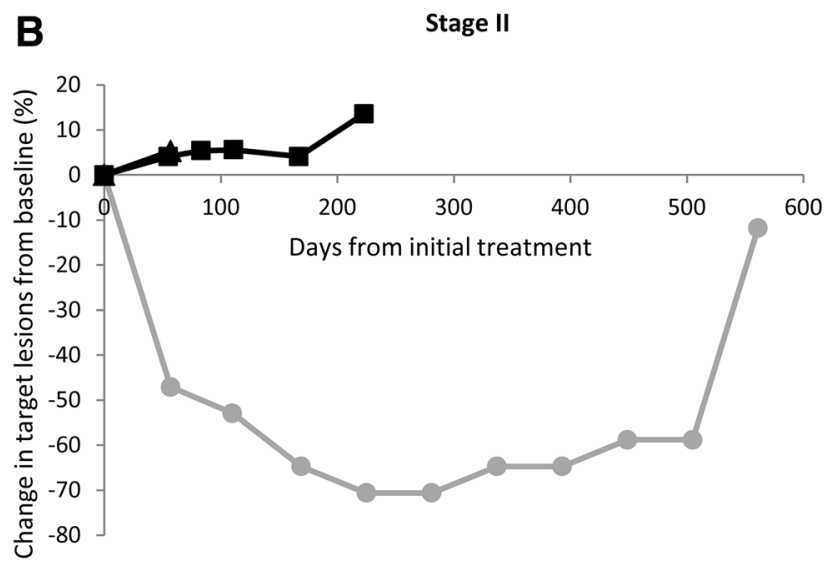

IPAT $200 \mathrm{mg}+\mathrm{ABI}+\mathrm{PRE} \longrightarrow$ IPAT $400 \mathrm{mg}+\mathrm{ABI}+\mathrm{PRE}$

Best overall response: $\triangle P D \quad \square$ SD $\bigcirc \mathrm{CR}$

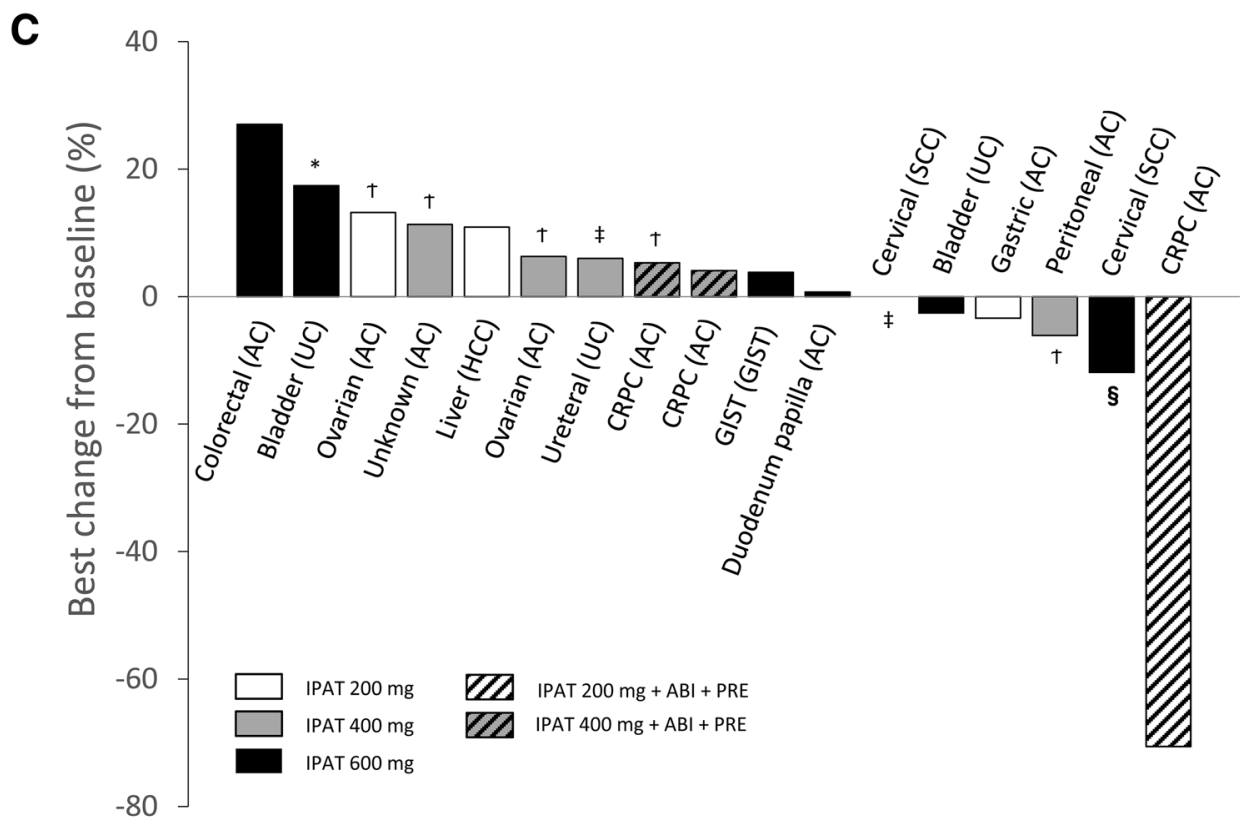

* PTEN loss and PIK3CA mutation; † PIK3CA mutation; ‡ PIK3CA amplification; § PIK3CA mutation and amplification; no symbol not detected or not evaluable

Fig. 3 Percentage change from baseline in tumor lesions during a Stage I $(n=14)$; b Stage II $(n=3)$; and c best percentage change from baseline in target lesions and PIK3CA mutation/amplification and PTEN loss. $A B I$ abiraterone, $A C$ adenocarcinoma, $C R$ complete response, $C R P C$ castration-resistant prostate cancer, GIST gastrointestinal stromal tumor, $H C C$ hepatocellular carcinoma, IPAT ipatasertib, $P D$ progressive disease, $P R E$ prednisolone, $S C C$ squamous cell carcinoma, $S D$ stable disease, $U C$ urothelial carcinoma reason for this increase in $\mathrm{AUC}_{0-24}$ is unknown. Although the pharmacokinetics of abiraterone in combination with ipatasertib were not analyzed in this study, they were assessed in a previous study [11] and the AUC and $C_{\max }$ of abiraterone were shown to be similar to that of abiraterone monotherapy (data not published).

PTEN loss and PIK3CA/AKT1 mutation/amplification have been studied as potential biomarkers for ipatasertib response in combination therapy. The A.MARTIN study in patients with mCRPC who were treated with ipatasertib plus abiraterone and prednisone/prednisolone showed that the combination increased radiographic PFS in patients with PTEN loss, indicating that PTEN loss may be a predictive biomarker of response [22].

The LOTUS study reported that ipatasertib in combination with paclitaxel improved PFS in patients with 
PIK3CA/Akt1/PTEN-altered TNBC, suggesting that PIK3CA/Akt1/PTEN alterations can also be biomarkers of response to ipatasertib in patients with breast cancer [18]. In the present study, tumor shrinkage was observed in two patients with PIK3CA mutation in the helical domain, which is a hotspot for PIK3CA mutations [23, 24]. However, none of the tumor samples in our cohort had Akt1 mutations or amplifications.

The genetic basis of prostate cancer is complex and our understanding is continually evolving [25]. A recent systematic review noted that, in addition to genetic alterations in the PIK3CA/Akt1/PTEN pathway, prognosis in prostate cancer may be associated with alterations in genes controlling DNA methylation, such as those at the glutathione S-transferase pi (GSTP1) and the familial protein 1 isoform A (RASSF 1A) loci, and in androgen regulation, such as TMPRSS 2 and $E R G$ [25]. In addition, the most common genetic mechanism of PTEN loss in prostate cancer is deletion of the $10 q 23$ locus, whereas inactivating mutations predominate in other cancers [26]. As well as inactivating PTEN, 10q23 loss may impair the expression of surrounding genes (including tumor suppressors), which may also affect outcomes and treatment responses in prostate cancer [26]. The results of large-scale studies, such as phase III studies, are needed to clarify which molecular biomarkers can act as prognostic indicators in patients receiving ipatasertib.

The main limitation of the present study was that potential biomarkers of tumor response to ipatasertib could not be determined due to the small patient population. In addition, because this trial focused on a small group of Japanese patients, it is unclear that the safety results of ipatasertib from this trial can be generalized to other ethnic groups. Large trials are required to confirm the safety and efficacy of ipatasertib, and to clarify the prognostic significance of genetic alternations in patients who are receiving ipatasertib.

As noted earlier, the number of patients who underwent genetic analysis in this study was too small to detect a relationship between the tumor response and PTEN expression or mutation/amplification of PIK3CA and Aktl. Another potential issue is the use of archival samples because these samples may not reflect the gene profile of tumor tissues at the time that response was evaluated. Among available samples in the current study, the rate of PTEN loss was $14 \%$, which is lower than in other reports in men with CRPC $(\sim 40 \%)[27,28]$. One reason may be that genetic alterations in the PIK3CA/Akt1/PTEN pathway are less frequent among Asian men with prostate cancer than among Caucasian men [29, 30]. Therefore, the prognostic value of these molecular alterations probably varies by ethnicity.

In conclusion, ipatasertib as a monotherapy (MTD, $600 \mathrm{mg} /$ day) and in combination with abiraterone plus prednisolone (MAD, $400 \mathrm{mg} /$ day) was safe and well tolerated in Japanese patients with advanced or recurrent refractory solid tumors. Currently, there are two ongoing global phase III studies for ipatasertib that include Japanese patients. These are examining ipatasertib in combination with abiraterone plus prednisone/prednisolone for patients with mCRPC (NCT03072238) [31] and ipatasertib in combination with paclitaxel for metastatic TNBC/ hormone receptor-positive breast cancer (NCT03337724) [32].

Acknowledgements The authors thank Nishad Parkar, PhD, and Catherine Rees of inScience Communications, Springer Healthcare for writing the outline and first draft of the manuscript, respectively. This medical writing assistance was funded by Chugai Pharmaceutical Co., Ltd.

Funding This study was funded by Chugai Pharmaceutical Co., Ltd.

\section{Compliance with ethical standards}

Conflict of interest TD has received research grant from Taiho, Novartis, Merck Serono, Astellas, MSD, Janssen, Boehringer Ingelheim, Takeda, Pfizer, Eli Lilly, Sumitomo Dainippon, Chugai, Kyowa Hakko Kirin, Daiichi Sankyo, Celegene, BMS, Abbvie, and Quintiles, and consultant fee from Eli Lilly, Chugai, Kyowa Hakko Kirin, MSD, Daiichi Sankyo, Amgen, Sumitomo Dainippon, and Taiho. YF has received research funding from Chugai for the current study, as well as research funding grants from AbbVie, AstraZeneca, BMS, Daiichi Sankyo, Eisai, Eli Lilly, Incyte, Merck Serono, MSD, and Novartis for other research. YF has also received fees from AstraZeneca, BMS, Novartis, and ONO for participating in advisory boards, and from BMS, ONO, and Taiho as a member of their speakers' bureau, outside of the submitted work. NM has received research grant from Bayer, AstraZeneca, Taiho, MSD, Janssen, Chugai, Eli Lilly, Sanofi, Astellas, Eisai, and Shionogi, and lecture fees from MSD, AstraZeneca, Eisai, Ono, Kissei, Sanofi, Takeda, Chugai, BMS, Novartis, Bayer, Janssen, and Pfizer. JT has received fee from Eisai as an employment medical advisor. SI has received research grant from BMS, Eli Lilly, Eisai, Chugai, Daiichi Sankyo, Novartis, Merck Serono, Bayer, and Otsuka, and lecture fees from Eli Lilly, Chugai, and Taiho. AT, CE, and SN are employees of Chugai Pharmaceutical Co., Ltd. ST has received research funding from Chugai for the current study, fees from Novartis, MSD, Eisai, Taiho, Chugai, Daiichi Sankyo, Bayer, and AstraZeneca for advisory work, and research grants from MSD, Eisai, Taiho, Chugai, Daiichi Sankyo, Bayer, AstraZeneca, and Quintiles, outside of the submitted work.

Ethical approval All procedures performed in studies involving human participants were in accordance with the ethical standards of the institutional and/or national research committee and with the 1964 Helsinki declaration and its later amendments or comparable ethical standards.

Informed consent Informed consent was obtained from all individual participants included in the study.

Open Access This article is distributed under the terms of the Creative Commons Attribution 4.0 International License (http://creativeco mmons.org/licenses/by/4.0/), which permits unrestricted use, distribution, and reproduction in any medium, provided you give appropriate credit to the original author(s) and the source, provide a link to the Creative Commons license, and indicate if changes were made. 


\section{References}

1. Porta C, Paglino C, Mosca A (2014) Targeting PI3K/Akt/mTOR signaling in cancer. Front Oncol 4:64. https://doi.org/10.3389/ fonc. 2014.00064

2. Thorpe LM, Yuzugullu H, Zhao JJ (2015) PI3K in cancer: divergent roles of isoforms, modes of activation and therapeutic targeting. Nat Rev Cancer 15(1):7-24. https://doi.org/10.1038/ nrc3860

3. Miller TW, Rexer BN, Garrett JT, Arteaga CL (2011) Mutations in the phosphatidylinositol 3-kinase pathway: role in tumor progression and therapeutic implications in breast cancer. Breast Cancer Res 13(6):224. https://doi.org/10.1186/bcr3039

4. Nagata Y, Lan K-H, Zhou X, Tan M, Esteva FJ, Sahin AA, Klos KS, Li P, Monia BP, Nguyen NT (2004) PTEN activation contributes to tumor inhibition by trastuzumab, and loss of PTEN predicts trastuzumab resistance in patients. Cancer Cell 6(2):117-127. https://doi.org/10.1016/j.ccr.2004.06.022

5. Manning BD, Cantley LC (2007) AKT/PKB signaling: navigating downstream. Cell 129(7):1261-1274. https://doi. org/10.1016/j.cell.2007.06.009

6. Bitting RL, Armstrong AJ (2013) Targeting the PI3K/Akt/mTOR pathway in castration-resistant prostate cancer. Endocr Relat Cancer 20(3):R83-R99. https://doi.org/10.1530/ERC-12-0394

7. Edlind MP, Hsieh AC (2014) PI3K-AKT-mTOR signaling in prostate cancer progression and androgen deprivation therapy resistance. Asian J Androl 16(3):378-386. https://doi. org/10.4103/1008-682x.122876

8. Carver BS, Chapinski C, Wongvipat J, Hieronymus H, Chen Y, Chandarlapaty S, Arora VK, Le C, Koutcher J, Scher H, Scardino PT, Rosen N, Sawyers CL (2011) Reciprocal feedback regulation of PI3K and androgen receptor signaling in PTENdeficient prostate cancer. Cancer Cell 19(5):575-586. https:// doi.org/10.1016/j.ccr.2011.04.008

9. Battelli C, Cho DC (2011) mTOR inhibitors in renal cell carcinoma. Therapy 8(4):359-367. https://doi.org/10.2217/thy.11.32

10. Costa RLB, Han HS, Gradishar WJ (2018) Targeting the $\mathrm{PI} 3 \mathrm{~K} / \mathrm{AKT} / \mathrm{mTOR}$ pathway in triple-negative breast cancer: a review. Breast Cancer Res Treat 169(3):397-406. https://doi. org/10.1007/s10549-018-4697-y

11. de Bono JS, De Giorgi U, Nava Rodrigues D, Massard C, Bracarda S, Font A, Arranz Arija JA, Shih KC, Radavoi GD, Xu N, Chan WY, Ma H, Gendreau S, Riisnaes R, Patel P, Maslyar DJ, Jinga V (2019) Randomized phase II study of Akt blockade with or without ipatasertib in abiraterone-treated patients with metastatic prostate cancer with and without PTEN loss. Clin Cancer Res 25(3):928-936. https://doi.org/10.1158/1078-0432.CCR-18-0981

12. LoRusso PM (2016) Inhibition of the PI3K/AKT/mTOR pathway in solid tumors. J Clin Oncol 34(31):3803-3815. https:// doi.org/10.1200/JCO.2014.59.0018

13. Blake JF, Xu R, Bencsik JR, Xiao D, Kallan NC, Schlachter S, Mitchell IS, Spencer KL, Banka AL, Wallace EM, Gloor SL, Martinson M, Woessner RD, Vigers GP, Brandhuber BJ, Liang J, Safina BS, Li J, Zhang B, Chabot C, Do S, Lee L, Oeh J, Sampath D, Lee BB, Lin K, Liederer BM, Skelton NJ (2012) Discovery and preclinical pharmacology of a selective ATP-competitive Akt inhibitor (GDC-0068) for the treatment of human tumors. J Med Chem 55(18):8110-8127. https://doi.org/10.1021/jm301024w

14. Lin J, Sampath D, Nannini MA, Lee BB, Degtyarev M, Oeh J, Savage H, Guan Z, Hong R, Kassees R, Lee LB, Risom T, Gross S, Liederer BM, Koeppen H, Skelton NJ, Wallin JJ, Belvin M, Punnoose E, Friedman LS, Lin K (2013) Targeting activated Akt with GDC-0068, a novel selective Akt inhibitor that is efficacious in multiple tumor models. Clin Cancer Res 19(7):17601772. https://doi.org/10.1158/1078-0432.CCR-12-3072
15. Yan Y, Serra V, Prudkin L, Scaltriti M, Murli S, Rodriguez O, Guzman M, Sampath D, Nannini M, Xiao Y, Wagle MC, Wu JQ, Wongchenko M, Hampton G, Ramakrishnan V, Lackner MR, Saura C, Roda D, Cervantes A, Tabernero J, Patel P, Baselga J (2013) Evaluation and clinical analyses of downstream targets of the Akt inhibitor GDC-0068. Clin Cancer Res 19(24):6976-6986. https://doi.org/10.1158/1078-0432.CCR-13-0978

16. Adis Insight (2018) Ipatasertib-Genentech. https://adisinsigh t.springer.com/drugs/800031868. Accessed 05 July 2018

17. Saura C, Roda D, Rosello S, Oliveira M, Macarulla T, PerezFidalgo JA, Morales-Barrera R, Sanchis-Garcia JM, Musib L, Budha N, Zhu J, Nannini M, Chan WY, Sanabria Bohorquez SM, Meng RD, Lin K, Yan Y, Patel P, Baselga J, Tabernero J, Cervantes A (2017) A first-in-human phase I study of the ATP-competitive AKT inhibitor ipatasertib demonstrates robust and safe targeting of AKT in patients with solid tumors. Cancer Discov 7(1):102-113. https://doi.org/10.1158/2159-8290.CD-16-0512

18. Kim SB, Dent R, Im SA, Espie M, Blau S, Tan AR, Isakoff SJ, Oliveira M, Saura C, Wongchenko MJ, Kapp AV, Chan WY, Singel SM, Maslyar DJ, Baselga J, investigators L (2017) Ipatasertib plus paclitaxel versus placebo plus paclitaxel as first-line therapy for metastatic triple-negative breast cancer (LOTUS): a multicentre, randomised, double-blind, placebo-controlled, phase 2 trial. Lancet Oncol 18(10):1360-1372. https://doi.org/10.1016/S1470 $-2045(17) 30450-3$

19. National Cancer Institute (2009) Common terminology criteria for adverse events (CTCAE), Version 4.0

20. Eisenhauer EA, Therasse P, Bogaerts J, Schwartz LH, Sargent D, Ford R, Dancey J, Arbuck S, Gwyther S, Mooney M, Rubinstein L, Shankar L, Dodd L, Kaplan R, Lacombe D, Verweij J (2009) New response evaluation criteria in solid tumours: revised RECIST guideline (version 1.1). Eur J Cancer 45(2):228-247. https://doi.org/10.1016/j.ejca.2008.10.026

21. Stephens C, Harnish E, Bowermaster R, Djalilvand A, Smith D, Kim D, Gendreau S, Del Valle E (2018) Development of a companion diagnostic assay for the detection of phosphatase and tensin (PTEN) protein loss and treatment with ipatasertib in metastatic castration-resistant prostatic cancer (mCRPC). Cancer Res 78(13 Suppl):4531. https://doi.org/10.1158/1538-7445.AM201 $8-4531$

22. De Bono JS, De Giorgi U, Massard C, Bracarda S, Nava Rodrigues D, Kocak I, Font A, Arija J, Shih K, Radavoi GD (2016) PTEN loss as a predictive biomarker for the Akt inhibitor ipatasertib combined with abiraterone acetate in patients with metastatic castration-resistant prostate cancer (mCRPC). Ann Oncol 27(suppl_6):vi243-vi265. https://doi.org/10.1093/annonc/mdw37 2.02

23. Dirican E, Akkiprik M, Ozer A (2016) Mutation distributions and clinical correlations of PIK3CA gene mutations in breast cancer. Tumour Biol 37(6):7033-7045. https://doi.org/10.1007/s1327 7-016-4924-2

24. Ligresti G, Militello L, Steelman LS, Cavallaro A, Basile F, Nicoletti F, Stivala F, McCubrey JA, Libra M (2009) PIK3CA mutations in human solid tumors: role in sensitivity to various therapeutic approaches. Cell Cycle 8(9):1352-1358. https://doi. org/10.4161/cc.8.9.8255

25. Perdomo HAG, Zapata-Copete JA, Sanchez A (2018) Molecular alterations associated with prostate cancer. Cent Euro J Urol 71(2):168-176. https://doi.org/10.5173/ceju.2018.1583

26. Poluri RTK, Audet-Walsh E (2018) Genomic deletion at 10q23 in prostate cancer: more than PTEN loss? Front Oncol 8:246. https ://doi.org/10.3389/fonc.2018.00246

27. Ferraldeschi R, Nava Rodrigues D, Riisnaes R, Miranda S, Figueiredo I, Rescigno P, Ravi P, Pezaro C, Omlin A, Lorente D, Zafeiriou Z, Mateo J, Altavilla A, Sideris S, Bianchini D, Grist E, Thway K, Perez Lopez R, Tunariu N, Parker C, Dearnaley 
D, Reid A, Attard G, de Bono J (2015) PTEN protein loss and clinical outcome from castration-resistant prostate cancer treated with abiraterone acetate. Eur Urol 67(4):795-802. https://doi. org/10.1016/j.eururo.2014.10.027

28. Tao DL, Bailey S, Beer TM, Foss E, Beckett B, Fung A, Foster BR, Guimaraes A, Cetnar JP, Graff JN, Eilers KM, Small EJ, Corless CL, Thomas GV, Alumkal JJ (2017) Molecular testing in patients with castration-resistant prostate cancer and its impact on clinical decision making. JCO Precis Oncol 1:1-11. https://doi. org/10.1200/PO.16.00067

29. Chen R, Ren S, Yiu MK, Fai NC, Cheng WS, Ian LH, Naito S, Matsuda T, Kehinde E, Kural A, Chiu JY, Umbas R, Wei Q, Shi X, Zhou L, Huang J, Huang Y, Xie L, Ma L, Yin C, Xu D, Xu K, Ye Z, Liu C, Ye D, Gao X, Fu Q, Hou J, Yuan J, He D, Pan T, Ding Q, Jin F, Shi B, Wang G, Liu X, Wang D, Shen Z, Kong X, Xu W, Deng Y, Xia H, Cohen AN, Xu C, Sun Y (2014) Prostate cancer in Asia: a collaborative report. Asian J Urol 1(1):15-29. https://doi.org/10.1016/j.ajur.2014.08.007

30. Zhong J, Rao J (2016) Epidemiological and molecular features of prostate cancer in Asian men living in Asian countries: implications in screen and management. Clin Oncol (Belmont) 1:1100
31. de Bono JS, Bracarda S, Chi K, Massard C, Olmos Hidalgo D, Sandhu S, Sternberg CN, Gendreau S, Xu N, Baney T, Maslyar D, Sweeney CJ (2017) Randomized phase III trial of ipatasertib vs. placebo, plus abiraterone and prednisone/prednisolone, in men with asymptomatic or mildly symptomatic previously untreated metastatic castrate-resistant prostate cancer (mCRPC). Ann Oncol 28(suppl_5):834TiP

32. Dent R, Kim S, Oliveira M, Isakoff S, Barrios C, O’Shaughnessy J, Lu X, Wongchenko M, Bradley D, Mani A, Baselga J, Turner N (2018) A pivotal randomized phase III trial evaluating ipatasertib (IPAT) + paclitaxel (PAC) for PIK3CA/AKT1/PTEN-altered advanced triple-negative (TN) or hormone receptor-positive HER2-negative (HR +/HER2-) breast cancer (BC). J Clin Oncol 36(15_suppl):IPATunity 130

Publisher's Note Springer Nature remains neutral with regard to jurisdictional claims in published maps and institutional affiliations. 